\title{
Renderings of the Adriatic Sea coastline on early modern age nautical charts in the context of their navigational applicability
}

\author{
Josip Faricic $^{\mathrm{a}, *}$, Tome Marelic ${ }^{\mathrm{b}}$ \\ ${ }^{a}$ University of Zadar, Department of Geography, Zadar, Croatia; email: jfaricic@unizd.hr \\ ${ }^{b}$ University of Zadar, Department of Geography, Zadar, Croatia; email: tmarelic@unizd.hr \\ * Corresponding author
}

Keywords: nautical chart, map, early modern age, Adriatic Sea

\begin{abstract}
:
Early Modern Age nautical charts can be considered as a source of knowledge, a means of navigation and a medium of communication. Given that these charts are intended primarily for seafarers, it is reasonable to question the extent of their usability for the planning and implementation of various navigation tasks. The aim of the research is to determine the level of applicability of early Modern Age charts showing the Adriatic Sea - a dynamic economic-geographical system that developed in a complex geomorphological-oceanographic basin and multicultural environment in which the interests of several European and Eurasian powers intertwined and clashed. In the analysis of these charts, the quantitative and qualitative aspects of their utilitarian value, i.e. their functionality, were also taken into account.

The sample of charts showing the Adriatic Sea is divided into three sets. The core set consists of nautical charts made in the period from approximately 1500 to 1800 , most of which were produced as printed charts. In addition to them, manuscript (portolan) charts, specific to the late Middle Ages and also manufactured during the first centuries of the Modern Age, were also included in the chart sample. The reason for this is the attempt to determine which geometrical, aesthetical and informational features were transferred from manuscript to printed charts. The sample has also been supplemented with a set of maps from the Modern Age which are not primarily intended as nautical charts, but which contain certain elements typical for nautical charts, such as compass roses. In terms of their coastline and island representations, those maps are probably a compound spatial information composite, built upon data that was - at least to some extent - extracted from the contemporary nautical charts, which will also be further investigated in this research.

The preliminary chart sample, which will probably be additionally supplemented in the later stages of this research, consists of 26 charts and maps; 5 portolan charts from the late Middle Ages, 17 manuscript (portolan) and printed charts from the early Modern Age, and 4 Modern Age geographic maps on which the Adriatic Sea is shown. In this preliminary phase, the charts were georeferenced using the modern nautical chart (in Mercator projection) as a reference map and applying a 4-parameter Helmert transformation. The georeferencing process was based on a uniform sample of 44 to 45 points per chart, with their locations standardized across the whole range of charts in order to enhance various metric comparisons between them. Preliminary results suggest that from the end of the $13^{\text {th }}$ to the end of the $18^{\text {th }}$ century, the accuracy of the Adriatic Sea coastline renderings on nautical charts (expressed as RMSE - root mean square error) did not increase. Also, the results showed that the angular composition of the Adriatic Sea coastline renderings on sample charts was increasingly deviating from the direction of the contemporary magnetic North (according to the paleomagnetic model CALS3K.4). Since those charts lack additional textual data on the magnetic variation, it could be interpreted as one of their fundamental functional shortcomings in comparison to later (modern) nautical charts based on vast and systematic hydrographical surveys that have been conducted from the early $19^{\text {th }}$ century.
\end{abstract}

Note: This research is part of the scientific project IP-2020-02-5339 Early Modern Nautical Charts of the Adriatic Sea: Information Sources, Navigation Means and Communication Media (NACHAS) funded by the Croatian Science Foundation. 